\title{
The Effects of Rehearsal on the Functional Neuroanatomy of Episodic Autobiographical and Semantic Remembering: A Functional Magnetic Resonance Imaging Study
}

\author{
Eva Svoboda and Brian Levine \\ Rotman Research Institute, Baycrest, Toronto, Ontario, Canada M6A 2E1
}

This study examined the effects of rehearsal on the neural substrates supporting episodic autobiographical and semantic memory. Stimuli were collected prospectively using audio recordings, thereby bringing under experimental control ecologically valid, naturalistic autobiographical stimuli. Participants documented both autobiographical and semantic stimuli over a period of $6-8$ months, followed by a rehearsal manipulation during the $3 \mathrm{~d}$ preceding scanning. During functional magnetic resonance imaging scanning, participants were exposed to recordings that they were hearing for the first, second, or eighth time. Rehearsal increased the rated vividness with which information was remembered, particularly for autobiographical events. Neuroimaging findings revealed rehearsal-related suppression of activation in regions supporting episodic autobiographical and semantic memory. Episodic autobiographical and semantic memory produced distinctly different patterns of regional activation that held even after eight repetitions. Region of interest analyses further indicated a functional anatomical dissociation in response to rehearsal and memory conditions. These findings revealed that the hippocampus was specifically engaged by episodic autobiographical memory, whereas both memory conditions engaged the parahippocampal cortex. Our data suggest that, when retrieval cues are potent enough to engage a vivid episodic recollection, the episodic/semantic dissociation within medial temporal lobe structures endure even with multiple stimulus repetitions. These findings support the multiple trace theory, which predicts that the hippocampus is engaged in the retrieval of rich episodic recollection regardless of repeated reactivation such as that occurring with the passage of time.

\section{Introduction}

In recent years, autobiographical memory (AM) paradigms have been increasingly used to address issues in human memory that cannot be addressed using standard laboratory materials. A key factor in many patient and functional neuroimaging studies of remote memory, especially those concerning the function of medial temporal lobe (MTL) structures, is the age of memory. It is axiomatic that recollection of autobiographical details, thoughts, and feelings diminish with memory age (Johnson et al., 1988; Rubin and Schulkind, 1997). Against the background of this retention function, certain events are exposed to repetition or rehearsal, which enhances factual and semantic representation and reduces novelty (Linton, 1975; Neisser, 1981). Such events are more readily accessible to consciousness despite their age (Galton, 1879).

Repetition or rehearsal effects bear on theories concerning involvement of the MTL structures in remote memory. Accord-

\footnotetext{
Received July 23, 2008; revised Dec. 10, 2008; accepted Jan. 15, 2009.

This work was supported by Canadian Institutes of Health Research doctoral award (E.S.) and Grant MGP-62963 (B.L.) and National Institute of Child Health and Human Development Grant HD42385-01 (B.L.). We thank Marina Mandic for help with image analysis and Adriana Restagno, Jovanka Skocic, Namita Kumar, and Irina Nica for their technical support. We are also grateful to Drs. Stefan Köhler and Morris Moscovitch for helpful comments during draft preparation.

Correspondence should be addressed to Brian Levine, Rotman Research Institute, Baycrest, 3560 Bathurst Street, Toronto, Ontario, Canada M6A 2E1. E-mail: blevine@rotman-baycrest.on.ca.

D01:10.1523/JNEUROSCI.3452-08.2009

Copyright $\odot 2009$ Society for Neuroscience $\quad$ 0270-6474/09/293073-10\$15.00/0
}

ing to the standard model of consolidation, the hippocampus has a time-limited role in both episodic and semantic memory such that long-term remote memories can be accessed directly via the neocortex (Squire et al., 1984; Squire and Alvarez, 1995). It also suggests that rehearsal of memories may facilitate this transitional process (Squire et al., 2004). Multiple trace theory (MTT) (Nadel and Moscovitch, 1997; Moscovitch et al., 2005) holds that the hippocampus has a time-limited role for semantic, but not episodic remote memory. Predictions arising from these theories are typically assessed by contrasting remote versus recent events without controlling for repetition or rehearsal effects. A fuller understanding of recency effects within remote memory requires the examination of the effects of memory repetition or rehearsal independently from memory age.

In studies of laboratory materials, repetition of novel associations between items and spatial contexts evokes suppression of hippocampal activation (Dolan and Fletcher, 1997; Binder et al., 2005; Köhler et al., 2005), whereas single-item repetition or familiarity strength evokes suppression of activation in anterior parahippocampal regions (i.e., perirhinal and entorhinal cortex) (Gonsalves et al., 2005; Köhler et al., 2005). Results from posterior parahippocampal regions (i.e., parahippocampal cortex) are more ambiguous, with evidence for response to both recollection and familiarity (Köhler et al., 2005; Eichenbaum et al., 2007).

Although repetition and rehearsal are assumed to facilitate accessibility of AMs, the neural correlates of this effect have been minimally assessed (Addis et al., 2004; Levine et al., 2004; Green- 
berg et al., 2005; Nadel et al., 2007). A major constraint on the assessment of rehearsal effects within AM is the use of retrospective collection of AM stimuli that are presumably already well rehearsed and therefore may strongly invoke semantic in addition to episodic mnemonic processes. As an alternative, prospective collection of events for later scanning (Cabeza et al., 2004; Levine et al., 2004) improves control over event selection, allowing experimental manipulation of rehearsal. In the present study, participants were scanned while listening to audio recordings selected from a pool of autobiographical events that they had created and that were assigned to different rehearsal conditions. Consistent with previous reports (for review, see Svoboda et al., 2006), we anticipated that the hippocampus, along with other regions supporting AM, would be preferentially engaged by recollection of these autobiographical events compared with semantic material. The critical question addressed by this study was how repetition would affect brain activation supporting episodic autobiographical and semantic memory. We expected repetition of autobiographical episodes to evoke suppression of the AM network, including the hippocampus. The endurance of preferential activation of the hippocampus for episodic AM despite the suppressive effect of rehearsal would support MTT, whereas reduction of hippocampal activation to baseline levels would be consistent with the standard model of consolidation.

\section{Materials and Methods}

Participants. Eleven well educated (mean education, 18 years; SD, 2.5 years) healthy adults (five men; six women), aged 24-42 (mean age, 30; $\mathrm{SD}, 5.34)$, participated in the study. All participants gave written informed consent for the study, which was approved by the hospital research ethics board.

Collection of autobiographical stimuli. Participants collected stimuli prospectively over $6-8$ months using a portable digital recorder (ICDBP100 V-O-R; Sony) following the methods specified by Levine et al. (2004). Extensive training on recording methodology was provided along with a detailed instruction manual and feedback on several practice recordings. A cue card was attached to each recorder for guidance.

There were two recording conditions, personal episodic (PE) and general semantic memory (GS). PE recordings comprised a 1-2 min description of a unique autobiographical episode, defined according to recent theoretical works on this topic (Wheeler et al., 1997; Tulving, 2002), including the story line, location, perceptions, thoughts, and emotional reactions. Participants were instructed to make PE recordings during or soon after the event occurred and within the same day (mean time elapsed since the event, $196 \mathrm{~min}$; SD, $132 \mathrm{~min}$ ). GS recordings comprised a 1-2 min reading from a book about neighborhoods in Toronto, Canada (excluding those evoking a specific autobiographical event), and were yoked in time to the PE recordings. Participants included a title in each recording (e.g., "Michael and Erika's wedding," "Bloor West Village"). $\mathrm{PE}$ recordings also included the time elapsed since the event and ratings for event uniqueness (1, routine; 4 , completely novel), personal importance ( 1 , not important; 4 , highly important), and emotional change as a result of the event (1, no change; 4 , major change). These ratings were used to match recordings across rehearsal conditions (see below). Participants were instructed not to listen to their recordings after dictating them.

On average, participants made 215 recordings across both conditions (range, 89-774). Of these, 62 recordings (an average of $46 \%$ of each individual's recordings; range, $8-69 \%$ ) were randomly selected for use in the study. This oversampling reduced the novelty of the recording activity as well as the predictability of which recordings would be used in the study. Although the act of recording may have changed the neural representation of the memory, behavioral research has shown that this has little effect on recall (Thompson, 1982). Accordingly, subjects did not report recollection of the habitual recording act itself, although the information in the recording was very effective in evoking recollection of the personal episodes (see analysis of ratings below).
Rehearsal. Ten PE and 10 GS recordings were pseudorandomly assigned to each of three levels of rehearsal frequency: one, two, and eight (i.e., PE1, PE2, PE8, and GS1, GS2, and GS8) such that all experimental conditions were matched for time since recording and encoding ratings. PE1 and GS1 recordings were heard for the first time in the scanner, PE2 and GS2 recordings were heard for the second time in the scanner, and PE8 and GS8 recordings were heard for the eighth time in the scanner. The selection of rehearsal frequency was guided by a desire to reflect high and low conditions of rehearsal and an exponential function known to operate within remote memory (Rubin and Schulkind, 1997).

The rehearsal pool was created by including one copy of the PE2 and GS2 recordings and seven copies of the PE8 and GS8 recordings. The resulting 160 recordings were edited to a maximum of $90 \mathrm{~s}$ in length and distributed across three rehearsal sessions with PE2 and GS2 heard for the first time the day before scanning and PE8 and GS8 heard three times on each of $3 \mathrm{~d}$ and $2 \mathrm{~d}$ before scanning, and once on the day before scanning. PE1 and GS1 recordings were only heard in the scanner. The first two sessions contained 60 recordings each, leaving 40 recordings for the final session. The rehearsal sessions occurred no less than 1 month after completion of stimulus collection, and each session was separated by $24 \mathrm{~h}$. Recordings were pseudorandomized within each session such that each repetition was separated by at least three other recordings, and no more than three recordings from a single condition were presented consecutively (Calfee, 1968; Madigan, 1969).

Each recording was preceded by its title, read in the examiner's voice. During each rehearsal session, participants were asked to reexperience the event (PE) or to think about the semantic information (GS). After the first presentation of each recording, participants rated on a 1-10 point scale how often the event was thought about or discussed since the time of the original recording ( 1 representing never and 10 representing two to four times per week). In the second and third rehearsal sessions, intersession naturalistic rehearsal frequency was determined by asking participants to indicate the number of times (0-1-2-3, etc.) that the event or information was thought about or discussed since it was last heard. All recordings were rated for episodic vividness ( 1 , "I can't recall the specific event but I know it happened because I made a recording," to 10, "My memory for this event is so vivid that I feel as though it is happening right now"). For GS recordings, participants rated their recollection of thoughts, feelings, or events associated with creating the recording. Finally, each rehearsal playlist contained one randomly interspersed recording of the examiner asking a yes or no question to be answered in the rating column to confirm attention to the information contained in the recordings; all of these questions were answered correctly.

Scanning. Recordings were edited to $30 \mathrm{~s}$ in length and pseudorandomized to six runs of $\sim 10 \mathrm{~min}$ in duration such that each condition was equally represented across runs and no one condition was repeated in sequence. Eight odd-number detection (OND) baseline tasks (Stark and Squire, 2001), comprising a $30 \mathrm{~s}$ stream of auditory random digits (1-9), were also evenly distributed across runs (the two final runs contained two OND tasks). The study design was a hybrid of event-related and block methods in that each $30 \mathrm{~s}$ recording was treated as a long event during which continuous reexperiencing or condition-related mental activity occurred. PE and GS recordings were in the participants' own voice; OND recordings and all recording titles were in the experimenter's voice. After auditory presentation of the recording title (or "Odd number detection"), participants were instructed to close their eyes and to mentally reexperience events (PE), to think about the semantic information (GS), or to mentally note odd numbers (OND). Auditory stimuli were presented in stereo format using acoustically padded functional magnetic resonance imaging (fMRI)-compatible headphones (Silent Scan; Avotec).

After each recording, participants opened their eyes and viewed the vividness rating scale (1-10), backprojected on a screen through angled mirrors mounted on the head coil. Ten seconds were allotted to rate reexperiencing via fMRI-compatible key pads (Lightwave Technologies). They were instructed to rate the degree of reexperiencing occurring in response to the recording (for the GS or OND conditions, this included intrusive episodic thoughts or episodic details from a rehearsal session). This was followed by a $5 \mathrm{~s}$ rest period, and then a tone signaling the 
participant to close their eyes and listen to the next title and recording. After the scan, PE1 and GS1 recordings were rated for frequency of naturalistic rehearsal since the time of the original recording.

fMRI data acquisition. A full-body 3 tesla MRI scanner (Signa EXCITE hardware; E2M4 software; GE Healthcare) with a standard quadrature birdcage transmit/receive head coil was used for scanning. The functional signal was measured from 28 slices ( $5 \mathrm{~mm}$ thick) with coronaloblique orientation that were graphically prescribed on the sagittal view and aligned perpendicular to the long axis of the hippocampus. Higher order shimming was used to reduce artifact in the anterior MTL. Six runs were obtained using a single-shot $\mathrm{T} 2^{*}$-weighted pulse sequence with spiral $k$-space readout [repetition time (TR), $2000 \mathrm{~ms}$; echo time (TE), 30 $\mathrm{ms}$; flip angle, $70^{\circ}$; field of view (FOV), $20 \times 20 \mathrm{~cm}$; effective acquisition matrix, $64 \times 64$; voxel size, $3.125 \times 3.125 \times 5 \mathrm{~mm}$; slice spacing, $0 \mathrm{~mm}$ ] (Glover and Lai, 1998). To allow magnetization to reach equilibrium, stimulus presentation was delayed by $20 \mathrm{~s}$ at the start of each experimental run. Reconstruction of the raw data was conducted off-line, and included gridding as well as correction for magnetic field distortion and Maxwell gradient terms.

Structural scans were acquired using three-dimensional T1-weighted fast spoiled gradient echo imaging (axial orientation, inversion recovery preparation, inversion time, $300 \mathrm{~ms}$; TR, $7.2 \mathrm{~ms}$; TE, $3.1 \mathrm{~ms}$; flip angle, $15^{\circ}$; effective acquisition matrix, $256 \times 192 ; 124$ slices; voxel size, $0.85 \times$ $0.85 \times 1.4 \mathrm{~mm}$; FOV, $22 \times 16.5 \mathrm{~cm}$ ) and were registered with functional images.

Data analysis. Behavior data (encoding, naturalistic rehearsal and scanning ratings, time lag between event occurrence and recording, age of recordings) were analyzed using repeated-measures ANOVA. Nonparametric analyses were used when data was not normally distributed.

Functional neuroimaging data preprocessing and analysis were performed using the Analysis of Functional Neuroimages software package (AFNI, version 20051230 0934) (Cox and Hyde, 1997). Functional time series data were corrected for rigid motion with spatial coregistration (maximum head movement did not exceed $3 \mathrm{~mm}$ in total). For every run, images acquired at each point in the series were aligned volumetrically to a reference image acquired during the scanning session. This reference image was selected based on calculations of a center reference value. Alignment parameters were then computed by an iterative weighted least-squares fit to the reference image and then applied using threedimensional Fourier transform interpolation. Physiological motions (respiration, cardiac pulsations), acquired using external sensors during scanning, were corrected with linear filtering. Spike motion was corrected by replacement with adjacent time points. The structural T1 acquisition scan was coregistered with functional time series data by finding the shift that maximized the overlap between the two modalities, using the automated AFNI $3 \mathrm{dAnatNudge} \mathrm{program}$. Overlap was visually inspected and manually corrected as necessary.

Each participant's functional time series data was submitted to deconvolution analysis. The input stimulus function and functional time series data were entered into the AFNI $3 \mathrm{dDeconvolve} \mathrm{program} \mathrm{to} \mathrm{estimate} \mathrm{the}$ impulse response function. Only epochs during the $30 \mathrm{~s}$ stimulus presentation were analyzed. A reference time series was created for the seven experimental conditions (PE1, PE2, PE8; GS1, GS2, GS8; OND). The impulse response function was then convolved on a voxel-by-voxel basis with the reference time series to attain an estimated hemodynamic response for each condition. Participants' activation maps were calculated for each condition relative to the OND baseline. Fifteen task contrast activation maps were specified. To facilitate group analysis, functional data for each task contrast were transformed into the Talairach coordinate system (Talairach and Tournoux, 1988) and spatially smoothed with a Gaussian filter of $6 \mathrm{~mm}$ full-width at half-maximum to account for individual variation of the anatomical landmarks and to increase the signal-to-noise ratio.

The effect of any absolute differences in the hemodynamic response across participants was removed by calculating a voxel-by-voxel percentage signal change relative to the OND baseline. The percentage change values for each condition and participant were entered into a voxelwise $3 \times 2 \times 11$ mixed-effects design ANOVA using rehearsal frequency ( 1,2 , and 8 ) and memory type (PE, GS) as the fixed factors and participants as
Table 1. Frequency of self-reported intersession naturalistic rehearsal across memory and rehearsal

\begin{tabular}{llll}
\hline Condition & Rehearsal 2 & Rehearsal 8 & Sum mean \\
\hline Personal episodic & $0.65(0.94)$ & $2.36(2.66)$ & $1.51(1.80)$ \\
General semantic & $0.07(0.15)$ & $0.91(1.74)$ & $0.49(0.95)$
\end{tabular}

SDs are displayed in parentheses beside the means. $1.00=1$ rehearsal.

Table 2. Mean vividness ratings across memory and rehearsal conditions

\begin{tabular}{lllll}
\hline Condition & Rehearsal 1 & Rehearsal 2 & Rehearsal 8 & Sum mean \\
\hline Personal episodic & $6.24(1.41)$ & $6.50(1.30)$ & $7.19(1.61)$ & $6.64(1.36)$ \\
General semantic & $1.93(0.91)$ & $2.48(1.36)$ & $2.55(1.31)$ & $2.32(1.10)$ \\
Odd number detection & & & & $0.14(0.45)$ \\
\hline
\end{tabular}

SDs are displayed in parentheses beside the means.

the random factor. A group statistical map was created for each experimental condition. $\alpha$ value and cluster size thresholds for group activation maps were determined using the AFNI AlphaSim program (Forman et al., 1995; Xiong et al., 1995). A corrected $p$ value of $0.05(p=0.002)$ and a minimum cluster size of $147 \mathrm{~mm}$ was used in the interpretation of imaging data.

In the second set of analyses, subregions in the MTL identified in the group activation maps were subjected to region of interest (ROI) analyses to examine the presence of a functional dissociation in response to $\mathrm{PE}$ and GS conditions. Each ROI comprised the peak activated voxel $(3.125 \times 3.125 \times 5 \mathrm{~mm})$, selected from individual PE versus OND activation maps across participants and entered into a within-subjects ANOVA (for similar methods, see Köhler et al., 2005).

\section{Results}

\section{Behavioral findings}

By design, qualitative event ratings taken at the time of recording (novelty, importance, emotionality) and the time lag between event occurrence and recording did not differ for recordings used across PE rehearsal conditions: $F_{(2,20)}=1.06, p=$ NS; $F_{(2,20)}=$ $0.880, p=\mathrm{NS}$, respectively; these data are not reported. Although naturalistic rehearsal of $\mathrm{PE}$ events between recording and experimental exposure was infrequent (frequency: $M, 2.82$; SD, 0.91), it was significantly greater than naturalistic rehearsal of GS events (frequency: M, 1.21; SD, 0.69) $(z=-2.85 ; p<0.005)$. For both $\mathrm{PE}$ and GS, greater experimental rehearsal was related to more intersession naturalistic rehearsal $\left(F_{(2,10)}=6.59\right.$; $\left.p<0.01\right)$ because of an intersession additive effect for the highest experimental rehearsal conditions (PE8 and GS8) presented over three sessions. There was no difference in intersession naturalistic rehearsal frequency for PE and GS recordings, resulting in equal naturalistic rehearsal exposure in both conditions $\left(F_{(1,8)}=3.53\right.$; $p=$ NS) (Table 1).

Vividness ratings were significantly higher for PE than GS recordings at initial exposure $\left(F_{(1,10)}=149 ; p<0.0001\right)$ (provided during rehearsal sessions or in the scanner for PE1 and GS1 conditions) and after completion of the rehearsal sessions $\left(F_{(1,10)}\right.$ $=56 ; p<0.0001$ ) (provided in the scanner). Overall, vividness ratings were highest for the PE condition, followed by the GS and OND conditions $\left(F_{(2,10)}=97 ; p<0.0001\right)$ (Table 2$)$. There was also an effect of rehearsal on vividness ratings across $\mathrm{PE}$ and GS conditions $\left(F_{(1.2,12.08)}=7.35 ; p<0.05\right)$, such that events were rated as more vivid with increasing repetition. This effect was more robust for the PE condition $\left(F_{(2,20)}=7.79 ; p<0.005\right)$ than the GS condition $\left(F_{(2,20)}=3.45 ; p=0.052\right)$ (Table 2$)$, although the memory condition by rehearsal interaction fell short of significance $(p=0.14)$. 
Table 3. Regional activation in response to episodic autobiographical memory rehearsal

\begin{tabular}{|c|c|c|c|c|c|c|c|c|c|}
\hline \multirow[b]{2}{*}{ Area of activation } & \multicolumn{3}{|l|}{ PE1 } & \multicolumn{3}{|l|}{ PE2 } & \multicolumn{3}{|l|}{ PE8 } \\
\hline & Coordinates & Tscore & Volume & Coordinates & Tscore & Volume & Coordinates & Tscore & Volume \\
\hline \multirow[t]{2}{*}{ Medial PFC (BA 9) } & -175526 & 5.28 & 539 & & & & & & \\
\hline & 84131 & 5.71 & 4137 & & & & & & \\
\hline Medial PFC (BA 8) & -84537 & 5.68 & 535 & -74738 & 5.05 & 221 & & & \\
\hline Mid-DL PFC (BA 46) & -431620 & 6.88 & 1642 & & & & & & \\
\hline Superior DL PFC (BA 6) & -41345 & 4.22 & 265 & & & & & & \\
\hline Anterior cingulate (BA 24, 32) & 191733 & 5.27 & 378 & & & & & & \\
\hline \multirow[t]{2}{*}{ Posterior cingulate (BA 31, 23, 30) } & $-13-5512$ & 9.81 & $10,423^{a}$ & $-7-5415$ & 6.09 & $987^{a}$ & $-15-4426$ & 7.13 & 286 \\
\hline & $8-5020$ & 8.10 & $10,423^{a}$ & $2-5221$ & 4.23 & $987^{a}$ & $6-4821$ & 6.92 & 3357 \\
\hline \multirow[t]{2}{*}{ Hippocampus } & $-35-34-1$ & 7.98 & $16,842^{a}$ & & & & & & \\
\hline & $-31-17-8$ & 5.87 & $16,842^{a}$ & & & & & & \\
\hline Temporal pole (BA 21/38) & $-546-19$ & 6.91 & $16,842^{a}$ & & & & & & \\
\hline \multirow[t]{2}{*}{ Superior temporal gyrus (BA 41) } & $-41-338$ & 10.84 & $16,842^{a}$ & $-44-337$ & 7.08 & 4690 & $-37-3311$ & 7.48 & 3005 \\
\hline & $36-289$ & 6.33 & $671^{a}$ & & & & & & \\
\hline Insula & $30-2812$ & 5.57 & $671^{a}$ & $37-257$ & 5.21 & 275 & $31-2710$ & 5.66 & 345 \\
\hline \multirow[t]{2}{*}{ Parahippocampus } & $-29-29-13$ & 5.31 & 271 & & & & & & \\
\hline & $17-35-10$ & 4.90 & 190 & & & & & & \\
\hline Superior temporal gyrus (BA 22) & $55-4-3$ & 8.84 & 1921 & $56-4-3$ & 7.47 & 1311 & $54-5-5$ & 7.31 & 1147 \\
\hline Temporoparietal junction (BA 22/39) & $-34-6216$ & 11.77 & 2253 & & & & & & \\
\hline Occipital lobe (BA 18, 19) & $-6-71-3$ & 4.92 & 422 & & & & & & \\
\hline \multirow[t]{2}{*}{ Thalamus } & $-2-81$ & 4.95 & 652 & & & & & & \\
\hline & $12-17$ & 4.40 & 223 & & & & & & \\
\hline \multirow[t]{2}{*}{ Caudate } & -101017 & 4.99 & 512 & & & & & & \\
\hline & 101419 & 5.03 & 606 & & & & & & \\
\hline \multirow[t]{4}{*}{ Cerebellum } & $-8-44-36$ & 6.38 & 4577 & $-24-29-41$ & 7.67 & 186 & & & \\
\hline & $-30-31-23$ & 6.25 & 178 & & & & & & \\
\hline & $-11-64-40$ & 4.41 & 216 & & & & & & \\
\hline & $25-27-22$ & 6.73 & 690 & & & & & & \\
\hline
\end{tabular}

$\mathrm{BA}$, Brodmann area; PFC, prefrontal cortex; DL, dorsolateral.

${ }^{a}$ Indicates shared activation cluster at corrected value of $p<0.05$. OND is the reference condition for each level of PE rehearsal $(1,2$, and 8$)$. For all tables, coordinates with cluster volumes $>147 \mathrm{~mm}$ and a $T$ score value $>4.13$ are corrected for multiple comparisons (corrected value of $p=0.05$ ).

\section{Neuroimaging findings}

Unrehearsed autobiographical remembering (PE1) activated a broad medial and left-lateralized network of regions consistent with findings of previous AM imaging studies (Maguire, 2001; Svoboda et al., 2006) and replicating our previous study with the prospective recording paradigm (Levine et al., 2004). Activated regions included the medial and lateral prefrontal cortices, anterior and posterior cingulate cortices, hippocampus, parahippocampal cortex, lateral and polar temporal cortices, temporoparietal junction, occipital cortex, thalamus, basal ganglia, and cerebellum. The overall pattern of brain activation was observed both when PE1 was contrasted with low-level baseline (odd number detection) (Table 3 ) and GS reference conditions (Table 4).

Rehearsal of PE resulted in global repetition suppression in regions activated during initial $\mathrm{PE}$ exposure (Fig. 1). Only the posterior cingulate, right insula, and bilateral lateral temporal cortices remained significantly activated after rehearsal (Table 3). This effect was mostly apparent at the PE2 condition, where only the medial prefrontal cortex was additionally active relative to the PE8 condition.

Unrehearsed GS information (GS1) activated a more restricted pattern of regions relative to OND than unrehearsed autobiographical remembering (PE1), including the posterior cingulate, lateral temporal, insular and parietal cortices, and hippocampus and parahippocampal cortex (Fig. 2, Table 5). These regions, with the exception of the parietal cortex, overlapped with a few regions activated in response to unrehearsed PE; however, engagement was more pronounced during PE (Table 4). Rehearsal of GS also resulted in repetition suppression. However, this effect was not apparent at GS2, where activations were stable or even increased (notably in the case of the posterior cingulate) relative to GS1 (Table 5). This may have occurred because of minimal recognition and associative processes during initial exposure to GS information when participants reported unfamiliarity with the material. Only bilateral lateral and polar temporal cortices as well as the right insula showed sustained activation at GS8.

$\mathrm{PE}$ and GS conditions were further contrasted to examine whether their activation patterns would continue to differ with rehearsal. Comparison of PE8 and GS8 (both conditions were additionally contrasted with OND baseline) revealed continued robust differences in overall regional activation, including bilateral medial prefrontal and left lateral inferior prefrontal cortices, left anterior and posterior cingulate, left parahippocampal cortex, lateral temporal cortex, temporoparietal junction, inferior parietal lobe, right caudate and bilateral cerebellum, suggesting that rehearsal alone does not reduce the episodic representation of PE relative to GS (Table 4).

To directly assess the effects of rehearsal on the medial temporal lobe components of the PE network at a greater level of sensitivity than possible from a whole-brain analysis, we conducted hypothesis-driven ROI analyses on peak signal from left anterior hippocampal and left parahippocampal cortex voxels activated in the PE condition. The anterior peak was selected because the anterior hippocampus is more commonly activated by AM than the posterior hippocampus, especially for recent as opposed to remote AMs (Gilboa et al., 2004; Svoboda et al., 2006).

This ROI analysis indicated a functional anatomical dissociation in response to rehearsal and memory conditions. A withinsubjects ANOVA revealed a significant three-way interaction between region (anterior hippocampus, parahippocampal cortex), memory condition (PE, GS), and rehearsal $(1,2,8)\left(F_{(2,20)}=\right.$ 
Table 4. Regional activation differentiating episodic autobiographical memory from general semantic memory

\begin{tabular}{|c|c|c|c|c|}
\hline \multirow[b]{2}{*}{ Area of activation } & \multicolumn{2}{|l|}{ PE1 vs GS1 } & \multicolumn{2}{|l|}{ PE8 vs GS8 } \\
\hline & Coordinates & $T$ score & Coordinates & Tscore \\
\hline \multirow[t]{2}{*}{ Medial PFC (BA 9, 10) } & -173821 & 8.06 & 45710 & 9.22 \\
\hline & 64434 & 5.95 & & \\
\hline Mid-DL PFC (BA 9) & -481625 & 5.80 & & \\
\hline \multirow[t]{2}{*}{ Superior medial PFC (BA 6) } & -71456 & 7.91 & -5757 & 6.59 \\
\hline & 12753 & 6.11 & & \\
\hline Inferior PFC (BA 47) & & & $-4117-14$ & 7.84 \\
\hline Anterior cingulate (BA 32) & -163424 & 8.55 & $441-7$ & 7.33 \\
\hline Posterior cingulate (BA 31) & $-7-5327$ & 8.36 & $-3-5130$ & 11.54 \\
\hline Hippocampus/lentiform & $-29-17-6$ & 4.92 & & \\
\hline Parahippocampus & & & $-23-34-13$ & 5.79 \\
\hline Superior temporal gyrus (BA 22) & & & $43-25-7$ & 9.79 \\
\hline Middle temporal gyrus (BA 21) & $-55-25-9$ & 7.39 & $-54-23-8$ & 10.67 \\
\hline \multirow[t]{2}{*}{ Temporoparietal junction (BA 39) } & $-34-6216$ & 9.70 & $-46-5517$ & 7.35 \\
\hline & & & $56-4710$ & 6.45 \\
\hline Temporal pole (BA 38/21) & $-585-14$ & 11.96 & & \\
\hline Inferior parietal lobe (BA 2) & & & $-40-2630$ & 5.32 \\
\hline Thalamus & $-5-1312$ & 6.63 & & \\
\hline Subthalamic nuclei & $-12-11-8$ & 6.23 & & \\
\hline \multirow[t]{2}{*}{ Caudate } & 51316 & 10.12 & 81117 & 7.10 \\
\hline & $36-416$ & 9.33 & -111013 & 7.41 \\
\hline Brainstem & & & $-1-26-10$ & 7.64 \\
\hline \multirow[t]{4}{*}{ Cerebellum } & $-30-50-49$ & 6.72 & $-30-49-38$ & 6.27 \\
\hline & $-37-72-24$ & 6.22 & & \\
\hline & $11-32-42$ & 8.49 & & \\
\hline & $18-62-45$ & 6.51 & & \\
\hline
\end{tabular}

BA, Brodmann area; PFC, prefrontal cortex; DL, dorsolateral.
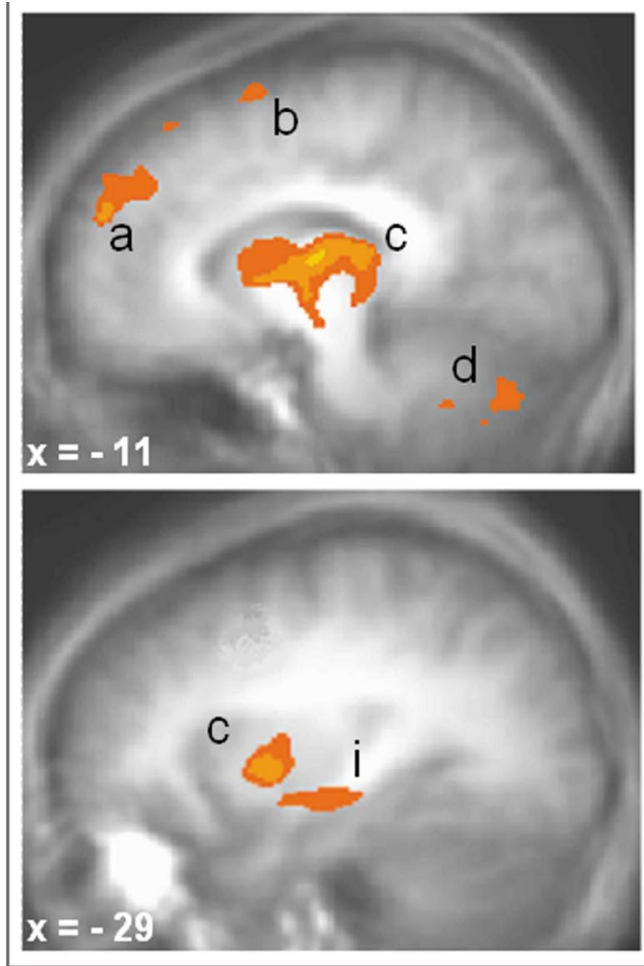
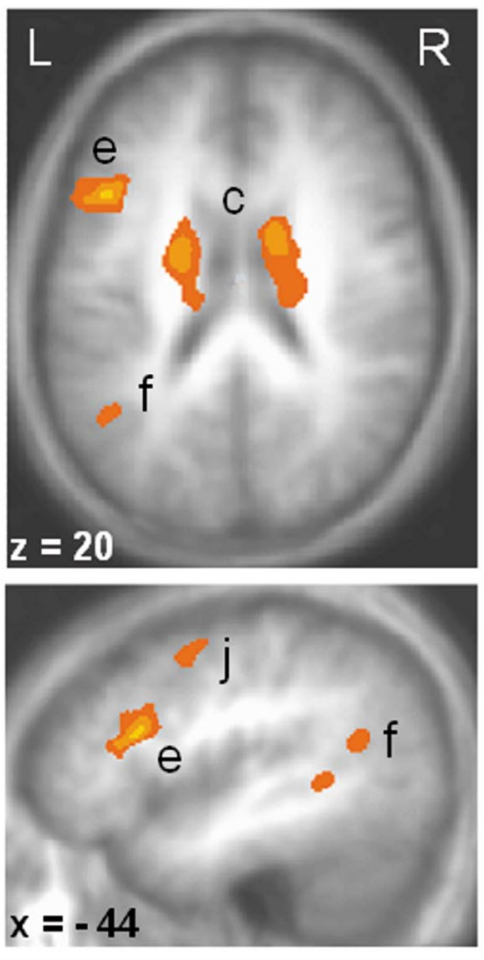
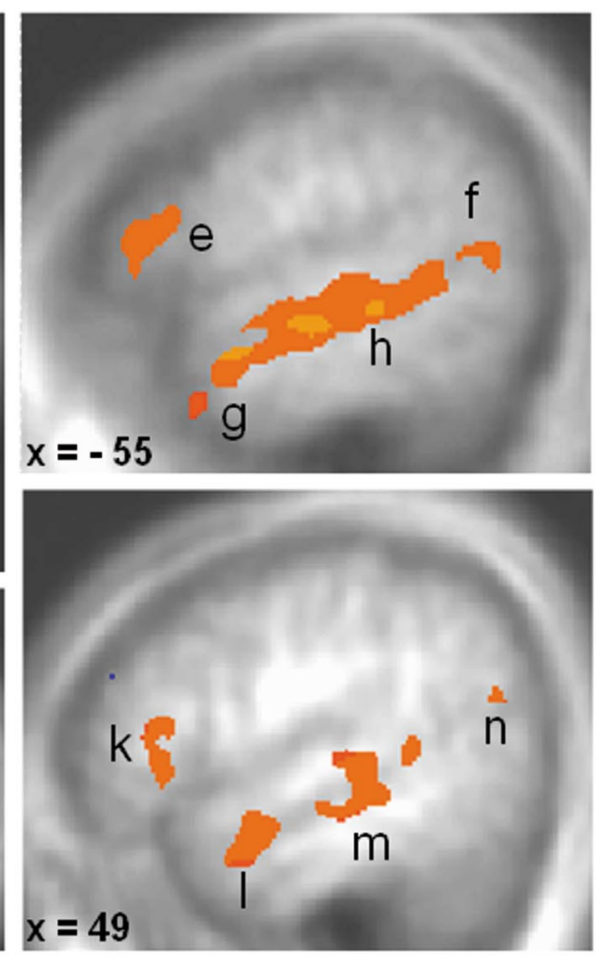

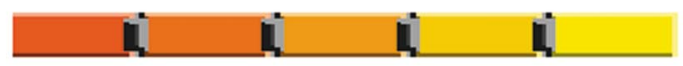

\subsection{3}

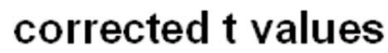

17.12

Figure 1. Regional activation in response to unrehearsed (PE1 vs OND baseline) relative to rehearsed (PE8 vs OND baseline): a, medial prefrontal cortex (PFC) [Brodmann area (BA) 9]; $b$, medial superior PFC (BA 6); c, thalamus spreading into lentiform; d, cerebellum; e, left mid-dorsolateral (DL) PFC (BA 46); f, temporoparietal junction (BA 39); $g$, temporal pole (BA 38); h, middle temporal gyrus (BA 21); i, hippocampus/lentiform; j, superior DL PFC (BA6); $k$, right mid-DL PFC (BA 46); I, right temporal pole (BA 38); m, superior temporal gyrus (BA 22/21); $n$, right temporoparietal junction (BA 39). Shown activation clusters are corrected for multiple comparisons ( $p=0.05$; cluster size $\geq 147 \mathrm{~mm}$ ). 

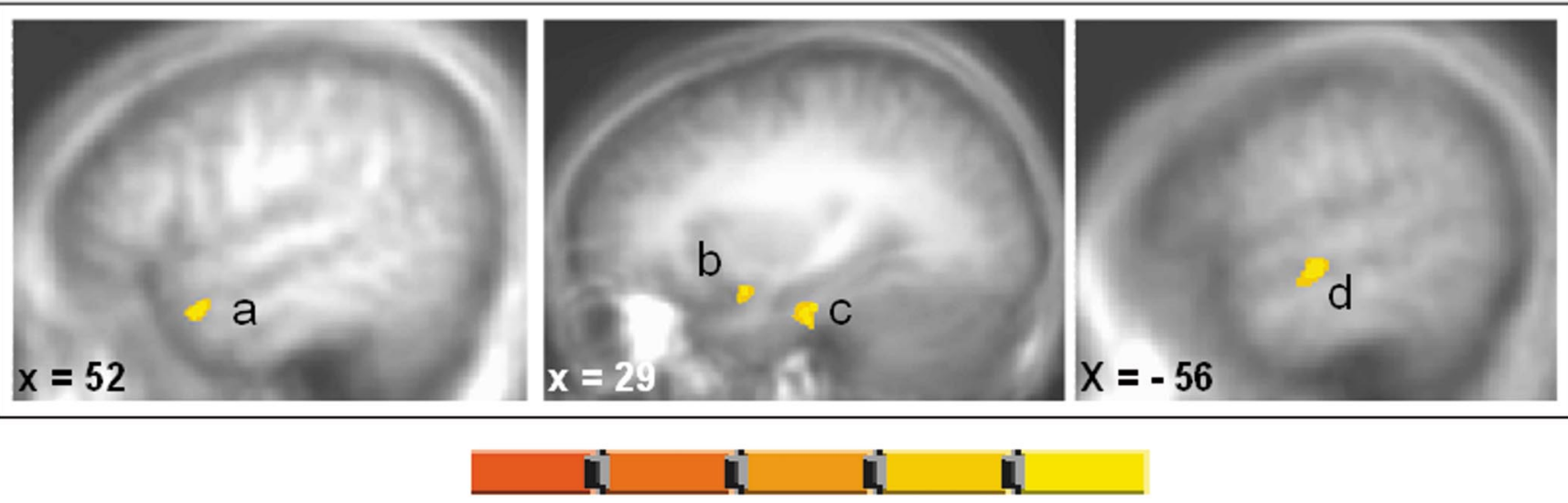

\subsection{3 corrected t values $\quad 6.04$}

Figure 2. Regional activation in response to unrehearsed (GS1 vs OND baseline) relative to rehearsed GS (GS8 vs OND baseline): a, temporal pole (BA 38); $b$, inferior PFC (BA 47); c, parahippocampal gyrus; and d, middle temporal gyrus (BA 21). Shown activation clusters are corrected for multiple comparisons ( $p=0.05$; cluster size $\geq 147$ mm). Activation in the hippocampus is not shown here (Table 5).

Table 5. Regional activation in response to general semantic memory rehearsal

\begin{tabular}{|c|c|c|c|c|c|c|c|c|c|}
\hline \multirow[b]{2}{*}{ Area of activation } & \multicolumn{3}{|l|}{ GS1 } & \multicolumn{3}{|l|}{ GS2 } & \multicolumn{3}{|l|}{ GS8 } \\
\hline & Coordinates & Tscore & Volume & Coordinates & Tscore & Volume & Coordinates & $T$ score & Volume \\
\hline \multirow[t]{3}{*}{ Posterior cingulate (BA 31, 23, 30) } & & & & $-14-5514$ & 6.19 & 1308 & & & \\
\hline & $12-5210$ & 6.94 & 3010 & $17-4910$ & 8.09 & $588^{a}$ & & & \\
\hline & $-28-25$ & & & $-30-29$ & & & & & \\
\hline \multirow[t]{2}{*}{ Hippocampus } & -10 & 5.97 & $1174^{a}$ & -10 & 5.42 & $588^{a}$ & & & \\
\hline & $-29-26$ & & & $-30-30$ & & & & & \\
\hline \multirow[t]{2}{*}{ Parahippocampus } & -13 & 6.29 & $1174^{a}$ & -12 & 6.39 & 1247 & & & \\
\hline & $17-34-10$ & 6.43 & $1600^{a}$ & $17-33-10$ & 7.80 & 1269 & & & \\
\hline Insula & $40-188$ & 9.00 & 5449 & $40-199$ & 5.72 & $6239^{a}$ & $38-2110$ & 5.33 & 294 \\
\hline Superior temporal gyrus (BA 41) & $-42-2811$ & 10.00 & $9059^{a}$ & $-37-3212$ & 7.08 & $6239^{a}$ & & & \\
\hline \multirow[t]{2}{*}{ Superior temporal gyrus (BA 22) } & $-55-172$ & 10.48 & $9059^{a}$ & $-56-154$ & 10.61 & $6239^{a}$ & $-55-134$ & 7.89 & 1511 \\
\hline & & & & & & & $62-86$ & 6.44 & $1049^{a}$ \\
\hline Temporal pole (BA 21/38) & & & & $58-4-3$ & 7.25 & 3371 & $60-4-4$ & 5.08 & $1049^{a}$ \\
\hline \multirow[t]{2}{*}{ Parietal lobe (BA 43, 1, 3) } & $-31-3067$ & 4.77 & 150 & & & & & & \\
\hline & $61-819$ & 7.16 & 391 & & & & & & \\
\hline \multirow{2}{*}{$\begin{array}{l}\text { Occipital lobe (BA 18) } \\
\text { Cerebellum (culmen) }\end{array}$} & & & & $48-71-5$ & 6.84 & 657 & & & \\
\hline & $23-27-20$ & 8.75 & $1600^{a}$ & & & & & & \\
\hline
\end{tabular}

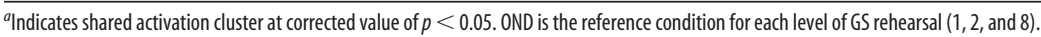

9.43; $p=0.001$ ) (Fig. 3). There was greater activation associated with PE than with GS in the left anterior hippocampus $\left(F_{(1,10)}=\right.$ $25.48 ; p=0.001)$, whereas activation in the left parahippocampal cortex was not modulated by memory condition $\left(F_{(1,10)}=0.86\right.$; $p=\mathrm{NS})$. The greater left anterior hippocampal activation for $\mathrm{PE}$ compared with GS endured across all levels of rehearsal, as indicated by a nonsignificant memory condition by rehearsal interaction $\left(F_{(2,20)}=2.51 ; p=\mathrm{NS}\right)$. Repetition suppression in the left anterior hippocampus and parahippocampal cortex was observed in response to both memory conditions $\left(F_{(2,20)}=39.69\right.$, $p<0.001$, and 9.81, $p=0.001$, for PE and GS, respectively). Pairwise tests indicated a significant stepwise reduction in left anterior hippocampal activation across all levels of PE, whereas repetition suppression for GS leveled off between GS2 and GS8. In the left parahippocampal cortex, activation leveled off between PE2 and PE8, whereas GS showed a stepwise effect significant at each pairwise contrast.

The anterior hippocampal peak from the PE1 versus OND contrast was selected for hypothesis-driven analyses. Nonetheless, there was also significant posterior hippocampal activation in this contrast $(x, y, z=-35,-34,-1)$ (Table 3). Ancillary ROI analyses were conducted to assess the effects of repetition on this voxel. As was the case for the anterior hippocampus, the posterior hippocampus from the PE1 versus OND contrast was more active in $\mathrm{PE}$ relative to $\mathrm{GS}\left(F_{(1,10)}=9.59 ; p<0.05\right)$, although not to the same degree as the anterior hippocampus $\left(F_{(1,10)}=34.91 ; p<\right.$ $0.001)$. The posterior hippocampal activation was not significantly modulated by rehearsal.

\section{Discussion}

Understanding the rehearsal effects on mnemonic stimuli is crucial to the understanding of the neural representation of remote memory, particularly in relation to the processing of episodic and semantic remote memories within the MTL. Assessment of rehearsal effects independent from age of memory is challenging if not impossible to accomplish using retrospectively collected autobiographical stimuli. In the present study, prospective collection of episodic autobiographical and semantic stimuli enabled the experimental manipulation of rehearsal while holding memory age constant, and controlling for other factors such as personal significance, emotion, and vividness known to modulate the neural correlates of AM retrieval (Svoboda et al., 2006). 


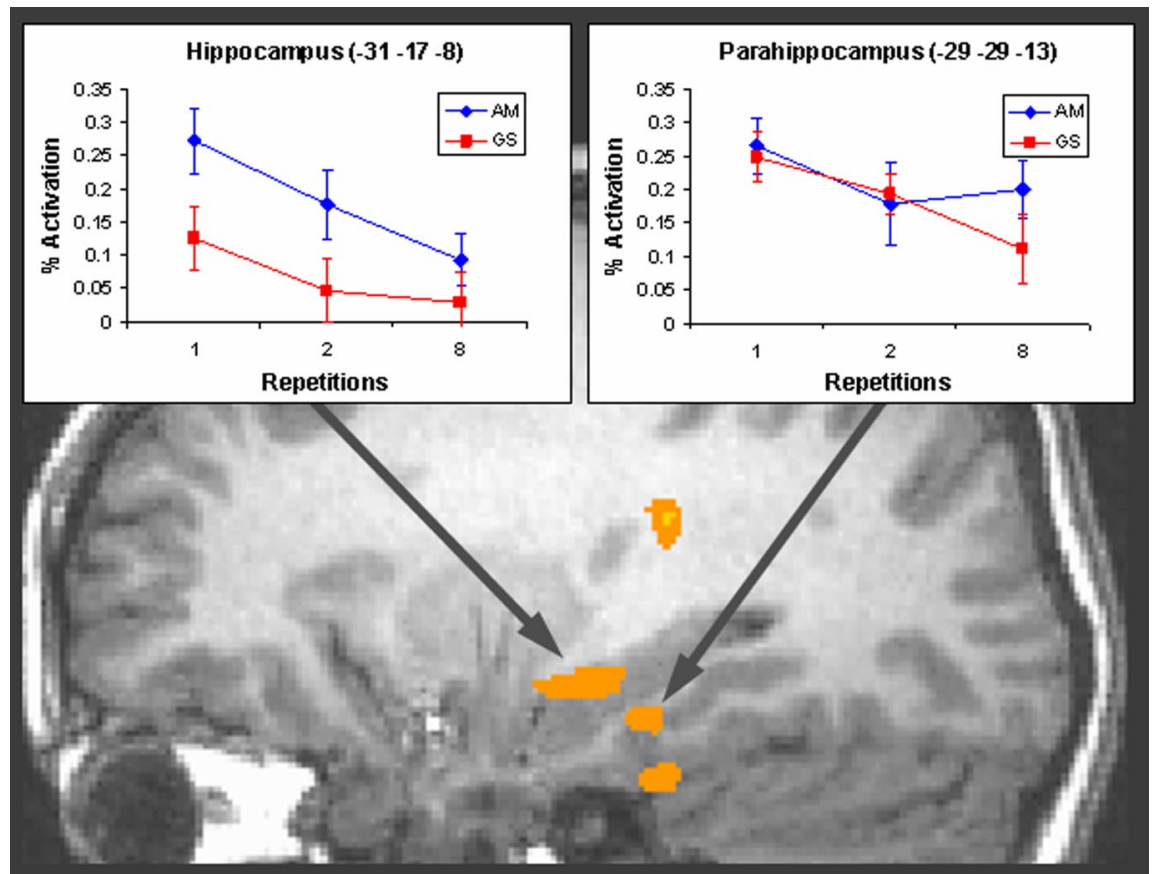

Figure 3. Peak activation voxels were extracted from activation clusters corrected for multiple comparisons ( $p=0.05$; cluster size $\geq 147 \mathrm{~mm}$ ) in the left anterior hippocampus and parahippocampal cortex corresponding to the PE1 versus OND activation map. The activation map was projected on a single subject's structural MRI image, seen here in a sagittal view $(x=-29)$. Graphs depict ROI for the anterior hippocampus and parahippocampal cortex activity across levels of experimental rehearsal and memory conditions. The simple effect of memory condition (PE vs GS) on anterior hippocampal activation was significant at all levels of experimental rehearsal, as depicted by asterisks on the left graph (values of $t_{(10)}=4.44,3.66$, and 2.40; values of $p \leq 0.001$, 0.005 , and 0.05 , for rehearsal 1,2 , and 8 , respectively). The simple effect of $P E$ rehearsal on anterior hippocampal activation was significant across all comparisons (values of $t_{(10)}=3.76,6.72$, and 3.38; values of $p \leq 0.005,0.001$, and 0.01 for 1 vs 2 , 1 vs 8 , and 2 vs 8 , respectively), whereas the simple effect of GS rehearsal was significant for comparisons 1 versus 2 and 1 versus 8 (values of $t_{(10)}=2.96$ and 4.19 ; values of $p<0.05$ and 0.005 , respectively). In the parahippocampal region, the rehearsal effect on PE was significant for comparisons 1 versus $2\left(t_{(10)}=2.52 ; p<0.05\right)$ and on $G S$ for comparisons 1 versus 8 and 2 versus 8 (values of $t_{(10)}=2.80$ and 2.34 ; values of $p<0.05$, respectively).

Autobiographical recollection of unrehearsed events activated a left lateralized and medial network of regions associated with autobiographical recollection and distinct from that associated with semantic memory (for review, see Maguire, 2001; Svoboda et al., 2006). As predicted from literature concerning stimulus repetition in priming and novelty detection (Habib, 2001; Henson, 2003), and several AM imaging studies involving repeated events (Addis et al., 2004; Levine et al., 2004; Greenberg et al., 2005) (but see Nadel et al., 2007), rehearsal attenuated activation in both episodic autobiographical and semantic memory networks. For PE, this was the case after even a single repetition. Despite this general repetition suppression, the pattern of activation associated with PE remained distinct from that associated with GS even after eight repetitions, supporting phenomenological qualities of recollection as rated by participants, which even increased with rehearsal.

As in this study, Nadel et al. (2007) found that rehearsal of retrospectively collected, self-selected, remote (i.e., decades old) memories increased the richness of AM recollection. Contrary to our findings, Nadel et al. (2007) found that rehearsal was associated with increased neocortical activation; hippocampal activation for rehearsed memories was comparable across high and low rehearsal conditions. The increase in neocortical activation was attributed to enhanced AM network activity elicited by repeated generative reconstruction of very remote events, as contrasted to the verbatim repetition of audio recordings of comparatively very recent and previously unrehearsed events that induced repetition suppression in the present study. Despite the major differences across these two studies, the implications for MTL function are similar in that both showed that hippocampal activation associated with episodic AM is maintained across rehearsal conditions. These findings appear to be in contradiction to predictions of the standard model of consolidation in which rehearsal of memories is thought to facilitate their transition from hippocampal dependency to direct accessibility via the neocortex (Squire et al., 2004).

Region of interest analyses suggested that the hippocampus is specifically engaged by episodic AM. Crucially, the greater left hippocampal activation for episodic relative to semantic memory endured despite the suppression in this region with repetition of episodic autobiographical memories. There is strong evidence that the hippocampus is centrally involved in binding of disparate elements for recollection, whereas extrahippocampal MTL structures are engaged by processes reliant on familiarity (Brown and Aggleton, 2001; Eichenbaum et al., 2007) (but see Squire et al., 2007). The binding function is inherent to the richness of event representation in naturalistic episodic AM. Our data suggest that when retrieval cues are potent enough to engage a vivid episodic recollection, the episodic/semantic dissociation within MTL structures endures even with multiple stimulus repetitions. These findings support MTT, which predicts a positive relationship between hippocampal function and episodic richness regardless of the passage of time or amount of mnemonic reactivations. The parahippocampal region was responsive to remote memory in general, possibly reflecting the functional heterogeneity of this region (Köhler et al., 2005).

Although activation in response to AM was observed in both anterior and posterior aspects of the hippocampus, ROI analyses further showed that sensitivity to rehearsal was significantly more robust in the anterior hippocampus. In a similar vein, Gilboa et al. (2004) found that recent AM events (which are relatively novel and have presumably undergone fewer lifetime rehearsals) activated the anterior axis, whereas remote AM events (which are older and have presumably undergone a greater number of lifetime rehearsals) showed a wider distribution of activation along the anterior-posterior axis. The few AM imaging studies that have found greater hippocampal activation in response to new relative to older events also showed activation in the anterior-to-mid aspects of the hippocampus (Niki and Luo, 2002; Maguire and Frith, 2003; Piefke et al., 2003). Moreover, episodic memory studies using laboratory stimuli have suggested that the anterior hippocampus may play a role in the detection of novel stimuli (Henson et al., 2003; Binder et al., 2005; Daselaar et al., 2006) or novel associations (Dolan and Fletcher, 1997; Binder et al., 2005; Köhler et al., 2005). Our findings suggest that, although 
the hippocampus continues to bind events over repeated recollections, this process may be differentially engaged along the anterior-posterior axis.

Animal studies have suggested that the MTL may be involved in coding the relative familiarity or recency of stimuli (Fahy et al., 1993; Brown and Xiang, 1998; Xiang and Brown, 1998). This raises the interesting possibility of whether repetition suppression of neural activity in the MTL plays a role in long-term memory. Single-cell recordings in animals have only examined the effect of familiarity- or recency-based suppression up to a delay of $24 \mathrm{~h}$ (Fahy et al., 1993). A behavioral study in humans suggests a single-repetition priming effect lasting up to a year (Cave, 1997). The present AM findings used a 24 h delay after the final rehearsal session. To examine the biological feasibility of this suppressive effect in remote remembering, it would be necessary to introduce longer delays between rehearsal and scanning sessions. The paradigm used here also did not allow for the examination of recency and familiarity effects on MTL structures. The study by Greenberg et al. (2005), in which participants were scanned twice using the same AM paradigm, suggests that the effect of repetition suppression lasts for at least 3 weeks after initial exposure.

Repetition suppression in relation to AM was observed in regions that are inconsistently reported in the literature on functional neuroimaging of AM, including the hippocampus, mid-dorsolateral prefrontal cortex, and thalamus (Maguire, 2001; Svoboda et al., 2006). Suppression as a result of AM repetition likely affects the consistency with which these regions meet statistical thresholds across imaging studies. Notably, activation in the mid-dorsolateral prefrontal cortex is rarely observed in studies using typical retrospective methods for AM retrieval, but is reported in studies in which AM stimuli is prospectively collected (Cabeza et al., 2004; Levine et al., 2004), suggesting that the well rehearsed AM events that are used by necessity in retrospective AM studies place fewer demands on reconstructive and monitoring processes mediated by this region than prospectively collected events. Our findings also have implications for paradigms that use prescan interviews to collect events for later scanning (Maguire and Mummery, 1999). The effect of rehearsal or repetition induced by such interviews is likely to be related to the previous rehearsal exposure for that event. Events that have not been frequently rehearsed or repeated, as in this study, may be particularly sensitive to prescan interview effects, whereas highly rehearsed events may be relatively unaffected by the additional rehearsal induced by the prescan interview.

As observed in the PE condition, suppression of overall regional activation occurred in the GS condition by the eighth rehearsal. In contrast to $\mathrm{PE}$, however, activation related to the second GS rehearsal appeared to be more widespread and robust than to the first exposure. This effect, known as repetition enhancement (Desimone, 1996), has been observed in other studies of episodic memory, as well as in priming studies, in which authors have attributed it to a shift in the cognitive processes engaged during subsequent stimulus exposure (Schacter et al., 1996; Eldridge et al., 2000; Heckers et al., 2002). Similarly, priming studies that have manipulated unnamable objects (Schacter et al., 1995; Henson et al., 2000; James et al., 2002) or degraded stimuli (Malach et al., 1995; Dolan et al., 1997; George et al., 1999; Grill-Spector et al., 1999) have attributed enhanced activation to the formation of novel representations. Vividness ratings provided by our participants during scanning are consistent with this interpreta- tion. Participants in our study may have formed novel semantic representations by the second exposure to GS information after which suppression of activation in response to familiar representations occurred by the eighth exposure.

Although our goal was to assess rehearsal effects with ecologically valid stimuli, there are important caveats to the generalization of our findings. Our repetition manipulation induced mental rehearsal of events, not to be confused with repeated exposure to an actual event, which may have differential effects on the representation of that event (Smith, 1952; Linton, 1975; White, 2002). Although prospective collection of autobiographical stimuli improved control over selection of memories, different results may be obtained from more typical methods involving self-selection of remote, well established memories (Nadel et al., 2007). Naturalistic rehearsal or repetition does not occur in the same manner as induced by our rehearsal manipulation. For example, conversational recall of events is modulated by the speakers' goals, social factors, and instructions, which can affect accuracy (Tversky and Marsh, 2000; Marsh, 2007). Our goal was not to mimic naturalistic rehearsal but rather to induce low versus high rehearsal of verified events sufficient for hypothesis testing in a neuroimaging context. The memories used in this study are recent compared with other patient and neuroimaging studies in which the most recent memories may be years old (but see Rekkas and Constable, 2005; Hassabis et al., 2007). There is, however, justification for studying memories that are months, rather than years old given the exponential rate of forgetting in humans (Rubin and Schulkind, 1997) and the timescale of consolidation effects as observed in animal studies (Winocur et al., 2005). Finally, the effects of rehearsal or repetition beyond that induced by our manipulation are unknown. Under an assumption that repetition effects follow an exponential function, many more rehearsals (i.e., dozens) would be required to extend our conclusions. We suspect, however, that as long as the stimulus cue invokes binding operations, hippocampal activation will be maintained regardless of the number of rehearsals.

In conclusion, we examined the effects of rehearsal on the neural substrates supporting autobiographical and semantic memory. By using prospectively collected recordings, we attained a high degree of experimental control over ecologically valid, naturalistic autobiographical stimuli. Although only one rehearsal was required to induce repetition suppression of the autobiographical network, activation in this network was differentiated from semantic memory even after eight repetitions, supporting vividness of recollection reflected in participants' ratings. ROI analyses indicated that hippocampal activation associated with engagement of episodic autobiographical memory remained elevated relative to semantic memory even after eight rehearsals, suggesting that the hippocampus is preferentially engaged by contextually rich episodic memories over and above semantic memory even when the episodic memories are repeatedly reactivated, supporting MTT.

\section{References}

Addis DR, Moscovitch M, Crawley AP, McAndrews MP (2004) Recollective qualities modulate hippocampal activation during autobiographical memory retrieval. Hippocampus 14:752-762.

Binder JR, Bellgowan PS, Hammeke TA, Possing ET, Frost JA (2005) A comparison of two FMRI protocols for eliciting hippocampal activation. Epilepsia 46:1061-1070. 
Brown MW, Aggleton JP (2001) Recognition memory: what are the roles of the perirhinal cortex and hippocampus? Nat Rev Neurosci 2:51-61.

Brown MW, Xiang JZ (1998) Recognition memory: neuronal substrates of the judgement of prior occurrence. Prog Neurobiol 55:149-189.

Cabeza R, Prince SE, Daselaar SM, Greenberg DL, Budde M, Dolcos F, LaBar KS, Rubin DC (2004) Brain activity during episodic retrieval of autobiographical and laboratory events: an fMRI study using a novel photo paradigm. J Cogn Neurosci 16:1583-1594.

Calfee RC (1968) Interpresentation effects in paired-associate learning. J Verb Learn Verb Behav 7:1030-1036.

Cave CB (1997) Very long-lasting priming in picture naming. Psychol Sci 8:322-325.

Cox RW, Hyde JS (1997) Software tools for analysis and visualization of fMRI data. NMR Biomed 10:171-178.

Daselaar SM, Fleck MS, Prince SE, Cabeza R (2006) The medial temporal lobe distinguishes old from new independently of consciousness. J Neurosci 26:5835-5839.

Desimone R (1996) Neural mechanisms for visual memory and their role in attention. Proc Natl Acad Sci U S A 93:13494-13499.

Dolan RJ, Fletcher PC (1997) Dissociating prefrontal and hippocampal function in episodic memory encoding. Nature 388:582-585.

Dolan RJ, Fink GR, Rolls E, Booth M, Holmes A, Frackowiak RS, Friston KJ (1997) How the brain learns to see objects and faces in an impoverished context. Nature 389:596-599.

Eichenbaum H, Yonelinas AP, Ranganath C (2007) The medial temporal lobe and recognition memory. Annu Rev Neurosci 30:123-152.

Eldridge LL, Knowlton BJ, Furmanski CS, Bookheimer SY, Engel SA (2000) Remembering episodes: a selective role for the hippocampus during retrieval. Nat Neurosci 3:1149-1152.

Fahy FL, Riches IP, Brown MW (1993) Neuronal activity related to visual recognition memory: long-term memory and the encoding of recency and familiarity information in the primate anterior and medial inferior temporal and rhinal cortex. Exp Brain Res 96:457-472.

Forman SD, Cohen JD, Fitzgerald M, Eddy WF, Mintun MA, Noll DC (1995) Improved assessment of significant activation in functional magnetic resonance imaging (fMRI): use of a cluster-size threshold. Magn Reson Med 33:636-647.

Galton F (1879) Psychometric experiments. Brain 2:149-162.

George N, Dolan RJ, Fink GR, Baylis GC, Russell C, Driver J (1999) Contrast polarity and face recognition in the human fusiform gyrus. Nat Neurosci 2:574-580.

Gilboa A, Winocur G, Grady CL, Hevenor SJ, Moscovitch M (2004) Remembering our past: functional neuroanatomy of recollection of recent and very remote personal events. Cereb Cortex 14:1214-1225.

Glover GH, Lai S (1998) Self-navigated spiral fMRI: interleaved versus single-shot. Magn Reson Med 39:361-368.

Gonsalves BD, Kahn I, Curran T, Norman KA, Wagner AD (2005) Memory strength and repetition suppression: multimodal imaging of medial temporal cortical contributions to recognition. Neuron 47:751-761.

Greenberg DL, Rice HJ, Cooper JJ, Cabeza R, Rubin DC, Labar KS (2005) Co-activation of the amygdala, hippocampus and inferior frontal gyrus during autobiographical memory retrieval. Neuropsychologia 43:659-674.

Grill-Spector K, Kushnir T, Edelman S, Avidan G, Itzchak Y, Malach R (1999) Differential processing of objects under various viewing conditions in the human lateral occipital complex. Neuron 24:187-203.

Habib R (2001) On the relation between conceptual priming, neural priming, and novelty assessment. Scand J Psychol 42:187-195.

Hassabis D, Kumaran D, Maguire EA (2007) Using imagination to understand the neural basis of episodic memory. J Neurosci 27:14365-14374.

Heckers S, Weiss AP, Alpert NM, Schacter DL (2002) Hippocampal and brain stem activation during word retrieval after repeated and semantic encoding. Cereb Cortex 12:900-907.

Henson R, Shallice T, Dolan R (2000) Neuroimaging evidence for dissociable forms of repetition priming. Science 287:1269-1272.

Henson RN (2003) Neuroimaging studies of priming. Prog Neurobiol 70:53-81.

Henson RN, Cansino S, Herron JE, Robb WG, Rugg MD (2003) A familiarity signal in human anterior medial temporal cortex? Hippocampus 13:301-304.

James TW, Humphrey GK, Gati JS, Servos P, Menon RS, Goodale MA (2002)
Haptic study of three-dimensional objects activates extrastriate visual areas. Neuropsychologia 40:1706-1714.

Johnson MK, Foley MA, Suengas AG, Raye CL (1988) Phenomenal characteristics of memories for perceived and imagined autobiographical events. J Exp Psychol Gen 117:371-376.

Köhler S, Danckert S, Gati JS, Menon RS (2005) Novelty responses to relational and non-relational information in the hippocampus and the parahippocampal region: a comparison based on event-related fMRI. Hippocampus 15:763-774.

Levine B, Turner GR, Tisserand D, Hevenor SJ, Graham SJ, McIntosh AR (2004) The functional neuroanatomy of episodic and semantic autobiographical remembering: a prospective functional fMRI study. J Cogn Neurosci 16:1633-1646.

Linton M (1975) Memory for real-world events. In: Explorations in cognition (Norman DA, Rumelhart DE, eds), pp 376-404. San Francisco: Freeman.

Madigan SA (1969) Intraserial repetition and coding processes in free recall. J Verb Learn Verb Behav 8:828-835.

Maguire EA (2001) Neuroimaging studies of autobiographical event memory. Philos Trans R Soc Lond B Biol Sci 356:1441-1451.

Maguire EA, Frith CD (2003) Lateral asymmetry in the hippocampal response to the remoteness of autobiographical memories. J Neurosci 23:5302-5307.

Maguire EA, Mummery CJ (1999) Differential modulation of a common memory retrieval network revealed by positron emission tomography. Hippocampus 9:54-61.

Malach R, Reppas JB, Benson RR, Kwong KK, Jiang H, Kennedy WA, Ledden PJ, Brady TJ, Rosen BR, Tootell RB (1995) Object-related activity revealed by functional magneticresonance imaging in human occipital cortex. Proc Natl Acad Sci U S A 92:8135-8139.

Marsh EJ (2007) Retelling is not the same as recalling: implications for memory. Curr Dir Psychol Sci 16:16-20.

Moscovitch M, Rosenbaum RS, Gilboa A, Addis DR, Westmacott R, Grady C, McAndrews MP, Levine B, Black S, Winocur G, Nadel L (2005) Functional neuroanatomy of remote episodic, semantic and spatial memory: a unified account based on multiple trace theory. J Anat 207:35-66.

Nadel L, Moscovitch M (1997) Memory consolidation, retrograde amnesia and the hippocampal complex. Curr Opin Neurobiol 7:217-227.

Nadel L, Campbell J, Ryan L (2007) Autobiographical memory retrieval and hippocampal activation as a function of repetition and the passage of time. Neural Plast 2007:90472.

Neisser U (1981) John Dean's memory: a case study. Cognition 9:1-22.

Niki K, Luo J (2002) An fMRI study on the time-limited role of the medial temporal lobe in long-term topographical autobiographic memory. J Cogn Neurosci 14:500-507.

Piefke M, Weiss PH, Zilles K, Markowitsch HJ, Fink GR (2003) Differential remoteness and emotional tone modulate the neural correlates of autobiographical memory. Brain 126:650-668.

Rekkas PV, Constable RT (2005) Evidence that autobiographic memory retrieval does not become independent of the hippocampus: an fMRI study contrasting very recent with remote events. J Cogn Neurosci 17:1950-1961.

Rubin DC, Schulkind MD (1997) The distribution of autobiographical memories across the lifespan. Mem Cognit 25:859-866.

Schacter DL, Reiman E, Uecker A, Polster MR, Yun LS, Cooper LA (1995) Brain regions associated with retrieval of structurally coherent visual information. Nature 376:587-590.

Schacter DL, Alpert NM, Savage CR, Rauch SL, Albert MS (1996) Conscious recollection and the human hippocampal formation: evidence from positron emission tomography. Proc Natl Acad Sci U S A 93:321-325.

Smith ME (1952) Childhood memories compared with those of adult life. J Genet Psychol 80:151-182.

Squire LR, Alvarez P (1995) Retrograde amnesia and memory consolidation: a neurobiological perspective. Curr Opin Neurobiol 5:169-177.

Squire LR, Cohen NJ, Nadel L (1984) The medial temporal region and memory consolidation: a new hypothesis. In: Memory consolidation (Weingartner H, Parker E, eds), pp 185-210. Hillsdale, NJ: Lawrence Erlbaum Associates.

Squire LR, Stark CE, Clark RE (2004) The medial temporal lobe. Annu Rev Neurosci 27:279-306.

Squire LR, Wixted JT, Clark RE (2007) Recognition memory and the medial temporal lobe: a new perspective. Nat Rev Neurosci 8:872-883. 
Stark CE, Squire LR (2001) When zero is not zero: the problem of ambiguous baseline conditions in fMRI. Proc Natl Acad Sci USA 98:12760-12766.

Svoboda E, McKinnon MC, Levine B (2006) The functional neuroanatomy of autobiographical memory: a meta-analysis. Neuropsychologia 44:2189-2208.

Talairach J, Tournoux P (1988) Co-planar stereotaxic atlas of the human brain. Stuttgart, Germany: Thieme.

Thompson CP (1982) Memory for unique personal events: the roommate study. Mem Cognit 10:324-332.

Tulving E (2002) Episodic memory: from mind to brain. Annu Rev Psychol $53: 1-25$.

Tversky B, Marsh EJ (2000) Biased retellings of events yield biased memories. Cogn Psychol 40:1-38.
Wheeler MA, Stuss DT, Tulving E (1997) Toward a theory of episodic memory: the frontal lobes and autonoetic consciousness. Psychol Bull 121:331-354.

White R (2002) Memory for events after twenty years. Appl Cognit Psychol 16:603-612.

Winocur G, Moscovitch M, Fogel S, Rosenbaum RS, Sekeres M (2005) Preserved spatial memory after hippocampal lesions: effects of extensive experience in a complex environment. Nat Neurosci 8:273-275.

Xiang JZ, Brown MW (1998) Differential neuronal encoding of novelty, familiarity and recency in regions of the anterior temporal lobe. Neuropharmacology 37:657-676.

Xiong J, Gao JH, Lancaster JL, Fox PT (1995) Clustered pixels analysis for functional MRI activation studies of the human brain. Hum Brain Mapp 3:287-301. 